\title{
Preparation and Properties of a Novel Polymeric Damping Material
}

\author{
Yu, Chengbing \\ School of Materials Science and Engineering \\ Shanghai University \\ Shanghai, P .R. China \\ ycb101@shu.edu.cn \\ $\mathrm{Yu}, \mathrm{Xi}$ \\ School of Materials Science and Engineering \\ Shanghai University \\ Shanghai, P.R. China \\ zy_james06@163.com \\ Chen, Saisai \\ School of Materials Science and Engineering \\ Shanghai University \\ Shanghai, P .R. China \\ kevinchensai@163.com
}

\author{
Wu, Fangfang \\ School of Materials Science and Engineering \\ Shanghai University \\ Shanghai, P .R. China \\ 18801911531@163.com \\ Wang, Qi \\ School of Materials Science and Engineering \\ Shanghai University \\ Shanghai, P .R. China \\ 761648630@qq.com \\ Zhu, Wenteng \\ School of Materials Science and Engineering \\ Shanghai University \\ Shanghai, P .R. China \\ 475775637@qq.com
}

\begin{abstract}
Using structural materials with high damping property is known as one of the most effective method to solve vibration and noise problem at present, and polyacrylate is one of the most widely polymer damping materials. Polyacrylate was often used as a resin matrix in waterborne damping coating for its excellent weather resistance and oil resistance properties. However, conventional polyacrylate was narrow damping temperature range, which could not meet the requirements to apply practically. In the paper, Methyl methacrylate (MMA) and butyl acrylate (BA), ethyleneglycol dimethacrylate (EGDMA) and phosphate esters of peg monomethacrylate (PAM-100) were used to prepare three layers core-shell acrylate latex interpenetrating polymer networks (LIPN) via surface crosslinking emulsion polymerization. The structure and properties of the latex were characterized by DMA, DLS and AFM. Three layers core-shell acrylate LIPN with $1 w t \%$ EGDMA in both layer 1 and layer 2, 4wt \% in outmost layer, could be achieved excellent damping property, which the damping peak was high up to 1.12 and the damping temperature range $(\tan \sigma>0.3)$ was $90 \mathrm{oC}$, and had excellent adhesion to metal substrate and good film-forming property.
\end{abstract}

Keywords-damping; core-shell; LIPN; emulsion polymerization; damping

\section{INTRODUCTION}

The constructions of restaurant, recreation room, public spaces, cabins and medical room and other locations in ship are laid directly on the steel by deck covering at present, which is difficult to meet the requirements of International Maritime Organization (IMO) rules [1]. In order to meet the requirements of IMO rules, a modification of laying a damping coating between steel and deck covering is needed, so it is significant to study matrix resin which is a key component of coating.

Recently, using high damping materials is known as one of the most effective means to solve the vibration and noise problem [2-4], and polyacrylate is one of the most polymer damping materials used widely for its excellent weather resistance and oil resistance properties. However, conventional polyacrylate with narrow damping temperature range $\left(20^{\circ} \mathrm{C} \sim 30^{\circ} \mathrm{C}\right)$ could not meet the practical requirement well [4]. Meanwhile, the synthesis of core-shell latex and LIPN has attracted great attentions in the past few decades [5, 6], which have many applications in damping field for their special structure [710]. Therefore, molecular design is often used through interpenetrating network and multi-layer core-shell structure, which improves the damping performance of materials.

In our works, in order to improve the damping properties of conventional polyacrylate, suitable acrylate monomers, ethyleneglycol dimethacrylate (EGDMA) and phosphate esters of peg monomethacrylate (PAM-100), were used to prepare three layers core-shell acrylate LIPN by surface cross-linking emulsion polymerization, and the properties of these LIPNs were also studied.

\section{EXPERIMENTAL}

\section{A. Materials}

Methyl methacrylate (MMA) and butyl acrylate (BA), as two monomers, were purchased from Sinopharm Chemical Reagent Co. Ltd., and Vacuum distilled before use. Ethyleneglycol dimethacrylate (EGDMA), as a crosslinking agent, was purchased from Aladdin Industrial Corporation. Sipomer PAM100 (SPM-Rhodia), as a 
functional monomer, was used for anti-corrosion. Allyl group alcohol ether sulfate (NRS-10), as a polymerizable surfactant, was chosen and purchased from Shanghai Honesty Fine Chemical Co. Ltd. Lauryl alcohol polyoxyethylene sodium sulfate (CM-60), as an anionic surfactant, was purchased from Solvay. Ammonium persulfate (APS), as an initiator, was purchased from Sinopharm Chemical Reagent Co. Ltd. Sodium bicarbonate, as a buffering agent, was purchased from Sinopharm Chemical Reagent Co. Ltd. All chemicals were chemical pure, and distilled water was used in all experiments.

\section{B. Synthesis of three-layer core-shell IPN}

The synthesis routine was shown in Scheme 1. The core-shell polymerizations were conducted with a semicontinuous process. The first step involved was the synthesis of the core latex, which predetermined amount of EGDMA was added to a three-neck flask with MMA, BA, APS, CM-60, NRS-10 and water, and slowly stirred at $700 \mathrm{rpm}$ to make core pre-emulsion at $40^{\circ} \mathrm{C}$ for $30 \mathrm{~min}$. Then water, CM- 60 and proper amount of sodium bicarbonate were added into a four-neck flask as a base solution, followed by the addition of $20 \%(\mathrm{v} / \mathrm{v})$ of core pre-emulsion to the base solution. The next was to rise and keep at $82^{\circ} \mathrm{C}$ for $30 \mathrm{~min}$ in water bath to synthesize seed latex at the stirring rate of $250 \mathrm{rpm}$. Then the remains of core pre-emulsion were added with seed latex dropwisely within $1 \mathrm{~h}$. After that, the resulting emulsion was kept at $82^{\circ} \mathrm{C}$ for at least $1 \mathrm{~h}$ in order to finish the polymerization process, which latex 1 was obtained.

The two-layer latex was synthesized based on latex 1, which the latex was prepared in the previous steps to serve as seed latex. The aqueous solutions of APS, which was dissolved in water, MMA, BA and EGDMA were slowly added to latex 1 at $82^{\circ} \mathrm{C}$ about $1 \mathrm{~h}$. The emulsion polymerization was kept for at least $1 \mathrm{~h}$, which latex 2 was obtained.

The synthesis of latex 3 with three-layer structure was similar with that of latex 2. MMA, BA, APS which was dissolved in water and a predetermined amount of PAM100 were slowly added to latex 2 at $82^{\circ} \mathrm{C}$ for about $1 \mathrm{~h}$. After that, it was rise and kept at $88^{\circ} \mathrm{C}$ for $2 \mathrm{~h}$ to complete the reaction, which latex 3 with three-layer core-shell was prepared.

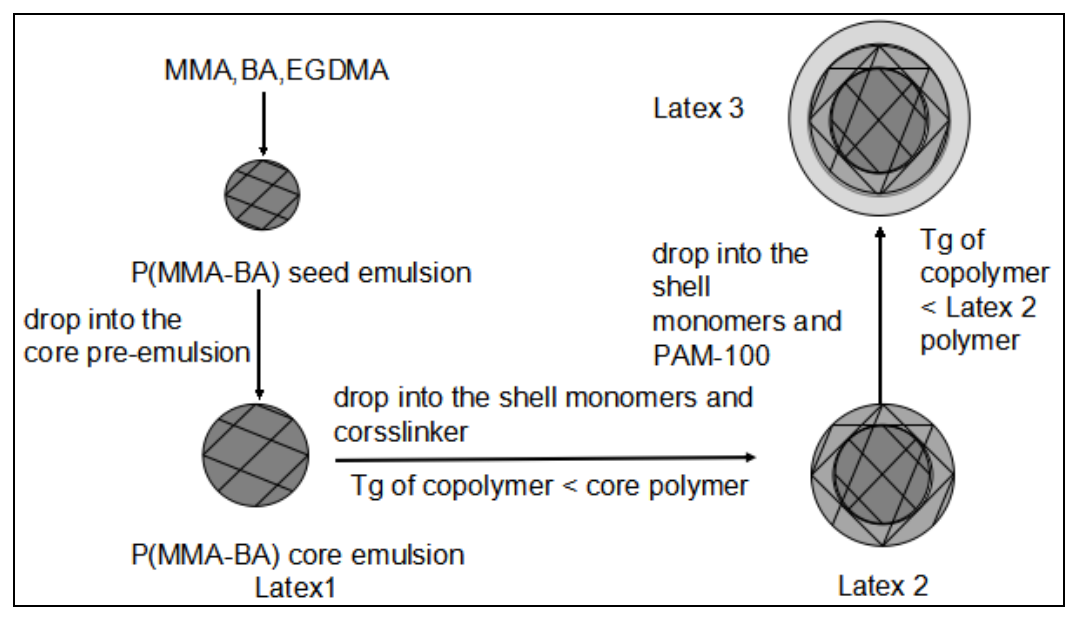

Scheme 1. Preparation route of three layers core-shell acrylate LIPN

\section{Characterization of Latex}

Damping properties analysis was performed by a DMA Q800 analyzer (TA Instruments) with tension film clamp over the temperature ranging from $-40^{\circ} \mathrm{C}$ to $100^{\circ} \mathrm{C}$ at a heating rate of $3^{\circ} \mathrm{C} / \mathrm{min}$ in $1 \mathrm{~Hz}$. The samples were rectangular bars $(15 \mathrm{~mm} \times 5.25 \mathrm{~mm} \times 0.8 \mathrm{~mm})$ and prepared by pouring the emulsion into a self-made template.

The emulsion was prepared on mica sheet by a high speed spin coating machine at $3000 \mathrm{rpm}$ for $15 \mathrm{~min}$. After that, the film was dried at room temperature for 1 day before AFM characterization. AFM characterization was carried out in a Digital Instruments Multimode Nanoscope, operating in peak force tapping mode.

The particle size and distribution (PSD) of synthesized emulsion were measured by a Malvern Zetasizer NanoZS90, and adhesion test was performed on QFZ type circle adhesion tester at $80-100 \mathrm{r} / \mathrm{min}$ to swing the rocking handle in clockwise direction.

\section{RESULTS AND DISCUSSION}

\section{A. Dynamic light scattering of latexes}

The dynamic light scattering analyses were performed with latexes 1-3, which were diluted to 1:100 with distilled water. The curves of size distribution in accordance with the number average were all similar in figure 1, all existing one peak. It was shown that all latex particles had a narrow size distribution, without noticeable new nucleation, which occurred in the whole polymerization process. 


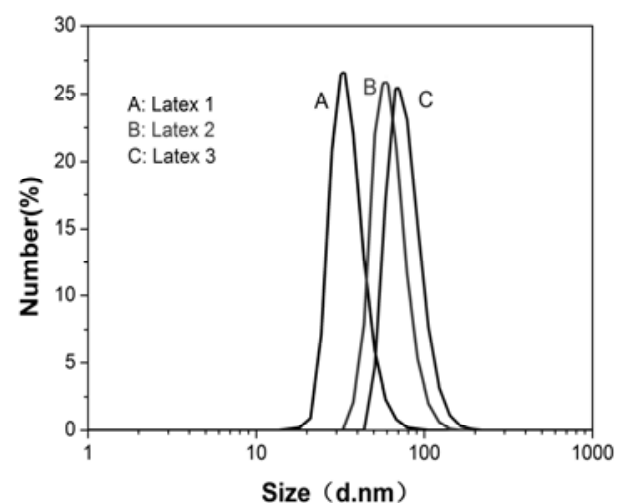

Figure 1. The particle size distributions (PSD) of three-layer latex particles

Polymerization process was important to three layers core-shell acrylate LIPN. It was the reason why the speed of feeding the second and third monomer mixtures to the seed latex was slower than that of initiator solution, which maintained the monomer concentration in the particles at a minimal value. The thickness of the first, second and third layers could be found and calculated as $61.27 \mathrm{~nm}, 20.71 \mathrm{~nm}$ and $18.35 \mathrm{~nm}$ from figure 1 , respectively. All these were illustrated that the monomers had polymerized to obtain the particles with layer-by-layer structure with step by step polymerization process, which increased the size of the latex particles.

\section{B. Effect of EGDMA content on damping properties}

Different amount of EGDMA were added in each layer, and the damping properties of three layers core-shell acrylate LIPNs were characterized by DMA, which were shown in figure 2. With increasing the amount of EGDMA from 0 to $1 \mathrm{wt} \%$, two narrow peaks was replaced by a broad peak, and the temperature range $(\tan \sigma>0.3)$ was widened further, the reason was why the IPN structure, which prevented the phase separation of components, was formed by the addition of EGDMA.

However, with increasing the amount of EGDMA further, the main peak was shifted to higher temperature, the value of damping peak was decreased, and the temperature range $(\tan \sigma>0.3)$ was narrower and narrower. All these were suggested that there existed an optimum EGDMA amount for three layers core-shell acrylate LIPN in terms of achieving the best damping properties, because the experiment was designed to synthesize the core with high glass transition temperature (Tg) first, then synthesized low $\mathrm{Tg}$ layer on the shell. Cross-linking structure of the cores was formed by the addition of EGDMA, which increased internal viscosity of latex particles, which inhibited the diffusion of adjacent outer monomers to the cores. When the cross-linking density was high enough, the segmental diffusion between the adjacent layers could be stopped, which was not beneficial to the formation of gradient interpenetrating structures, which could explain the narrow damping peak of the sample with $4 \mathrm{wt} \%$ EGDMA.

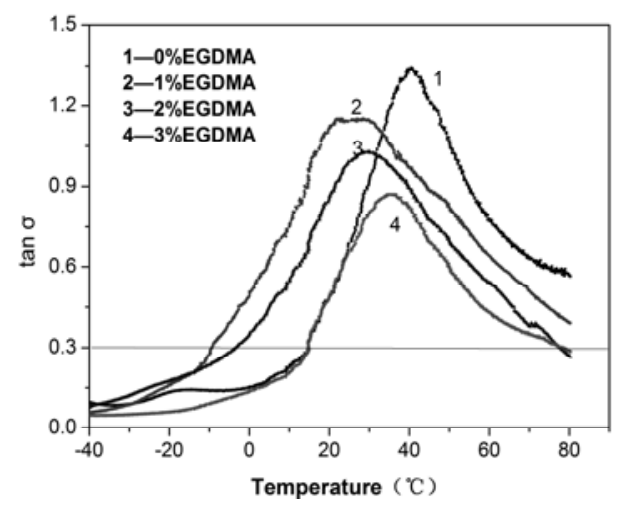

Figure 2. Effect of EGDMA on the damping properties of three layers core-shell acrylate LIPN

\section{Effect of PAM-100 content on the adhesion of coating films}

The effect of PAM-100 content on the adhesion of coating films was demonstrated in table1. From the table 1, + it was found out that, with the increasing of PAM-100 content, the adhesion on tinplate was improved from level 3 to level 1, due to the formation of RO (PO)O2Fe with the strong chelating between phosphate monoester and metal surface, which was a component of phosphating film to link firmly polymer with metal substrate through covalent bond. Therefore, the polymer coating and the metal substrate were linked closely by functional phosphate ester monomer in polymer backbone, which improved greatly the adhesion of the films.

TABLE 1. Effect of PAM-100 content on the adhesion of coating films

\begin{tabular}{cccccc}
\hline Item & \multicolumn{4}{c}{ PAM-100 content /\% (Account for shell monomer mass fraction) } \\
& 0 & 2 & 4 & 6 & 8 \\
\hline Adhesion / level & 3 & 2 & 1 & 1 & 1 \\
\hline
\end{tabular}

However, it was found in the experiment that, when the amount of PAM-100 in the system was more than $4 \mathrm{wt} \%$, the gel rate was augmented. It was known that, with the amount of PAM-100 increasing, $\mathrm{pH}$ value in polymerization system was decreased, which caused to demulsify in part. These were suggested that there existed an optimum PAM-100 amount for three layers core-shell acrylate LIPN in terms of achieving the best adhesive properties.

\subsection{The film morphology of three layers core-shell acrylate LIPN}

The morphology of films was formed at ambient temperature, which was characterized by AFM and it was shown in figure 3.The color line was represented height difference. As it was shown that, bright spots, which were formed by cross-linked hard core (layer 1 and layer 2), was dispersed in dark area, which was formed by soft shell 
(layer 3), and the maximum height difference of the latex film was $10 \mathrm{~nm}$ only according to the color line, which was shown that, the three layers core-shell acrylate LIPN had a good film forming property.

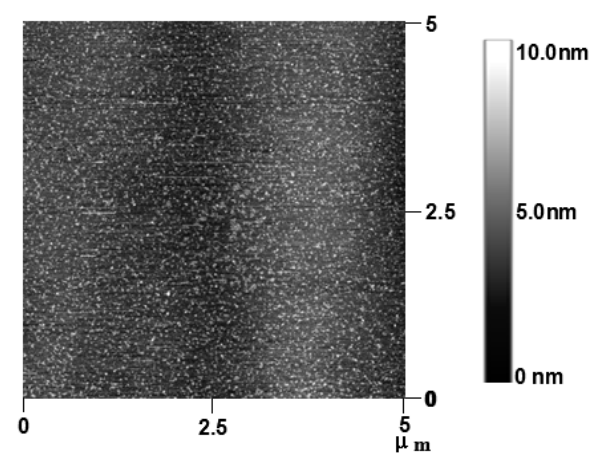

Figure 3. The AFM images of three layers core-shell acrylate LIPN

\section{CONCLUSIONS}

Three layers core-shell acrylate LIPN was prepared via surface cross-linking emulsion polymerization, which improved damping performance of acrylate emulsion. DLS showed that all the latex particles had a narrow size distribution. DMA showed that EGDMA content had a great influence on the damping properties of latex, with $1 \mathrm{wt} \%$ EGDMA in both layer 1 and layer 2 could achieve the best damping properties.

Adhesion test showed that the one polymerizing with $4 \mathrm{wt} \%$ PAM-100 could have an excellent adhesion to metal substrates. Moreover, three layers core-shell acrylate LIPN could form a continuous film at room temperature, which was confirmed by AFM. In conclusion, all were proved that the three layers core-shell acrylate LIPN had a potential application for waterborne damping coating for ship.

\section{ACKNOWLEDGEMENTS}

The work was financially supported by the Postgraduate Innovate Foundation of Shanghai University (2013-2015) and deeply gratitude was expressed to Instrumental Analysis \& Research Center of Shanghai University for use of their facilities.

\section{REFERENCES}

[1] The International Maritime Organization, " IMO IMO SOLAS and STCW rules take effect," International Cruise \& Ferry Review, Spring/Summer, 2010, pp. 81.

[2] Y.C. Liu, J.F. Li, G.C. Yang, and et al., "High damping functional materials prepared by spray deposition," Journal of Materials Processing Technology, vol. 106, 2000,pp. 94-98.

[3] S. B. Chen, Q. H. Wang, X. Q. Pei, and et al., “ Dynamic Mechanical Properties of Castor Oil-Based Polyurethane/Epoxy Graft Interpenetrating Polymer Network Composites," Journal of Applied Polymer Science, vol. 118, 2010, pp. 1144 -1151.

[4] N. Roche, M. N. Ichchou, Salvia, et al., “ Dynamic Damping Properties of Thermoplastic Elastomers Based on EVA and Recycled Ground Tire Rubber," Journal of Elastomers and Plastics, vol. 43, 2011, pp. 317-340.

[5] X. R. Chen, Y.Y. Wang, J.H. Zheng, and et al., "Development in polyacrylate damping materials," Aerospace Materials \& Technology, vol. 2 , 2004, pp. 7-25.

[6] X.L. Zhu, Q.S. Zhang, L.L. Liu, and et al., "Synthesis and characterization of a new compound bearing ketone and hydroxyl groups for preparation of ambient temperature selfcrosslinking waterborne

polyurethanes," Progress in Organic Coatings, vol. 59, 2007, pp. 324-330.

[7] A.B. Foster, P.A. Lovell, A. Lovell, and et al., "Control of adhesive properties through structured particle design of water-borne pressure-sensitive particle design of water-borne pressure-sensitive adhesives," Polymer,, vol. 50, 2009, pp. 16541670.

[8] X. Cheng, T. Qiu, J. Q. Deng, and et al., "Dynamic mechanical study on multilayer core-shell latex for damping applications," Progress in Organic Coatings, vol. 74, 2012, pp. 233-239.

[9] S. Li, W. Zeng, "Effect of crosslinker, buffer, and blending on damping properties of poly (styrene-acrylonitrile)/poly (ethyl acrylate-n-butyl acrylate) latex interpenetrating polymer networks," Journal of applied polymer science, , vol. 84, 2002, pp. 2347-2351.

[10] K. I. Suresh, S. Vishwanatham, E. Bartsch, "Viscoelastic and Damping Characteristics of Poly(n-butyl acrylate)-Poly(n-butyl methacrylate) Semi-IPN latex films," Polymers for Advanced Technologies, vol. 18, 2007, pp. 364-372. 